\title{
Efectos del traspaso inflacionario del tipo de cambio a la inflación: Canadá, Estados Unidos y México, 1990-2015
}

\section{The pass-through effects from exchange rate to inflation: Canada, the United States, and Mexico, 1990-2015}

\author{
IgnaCio Perrotini-Hernández * \\ Ruth Ortiz-Zarco *
}

\begin{abstract}
In this paper the pass-through effects from exchange rate fluctuations to inflation in the North American Free Trade Agreement (NAFTA) economies are analyzed. In the econometric estimation, causality relations between the interest rate and the exchange rate are empirically assessed for the cases of Canada, The United States, and Mexico. Furthermore, the role of the exchange rate in the inflation targeting monetary policy framework of the NAFTA economies is dealt with. It is shown that both Canada and The United States face a negligible pass-through coefficient, while Mexico's has declined along with inflation, although it remains positive.
\end{abstract}

Keywords: Pass-through, uncovered interest rate parity, inflation targeting.

\section{Resumen}

Analizamos el traspaso de los movimientos del tipo de cambio a la inflación (pass-through) para las economías del Tratado de Libre Comercio de América del Norte (TLCAN). En la estimación econométrica exploramos empíricamente las relaciones de causalidad entre la tasa de interés y el tipo de cambio de Canadá, Estados Unidos y México, así como el papel del tipo de cambio en el marco de política monetaria de metas de inflación (PMMI) de las tres economías. Nuestros resultados revelan que en Canadá y Estados Unidos de América el efecto de traspaso inflacionario es irrelevante, mientras que en México ha disminuido, aunque aún es significativo.

Palabras clave: traspaso inflacionario, paridad descubierta, metas de inflación. 


\section{Introducción}

El papel del tipo de cambio en el marco de política monetaria de metas de inflación (PMMI en adelante) ha sido objeto de múltiples análisis teóricos y empíricos. En el ámbito de la teoría pueden identificarse claramente dos posiciones: por una parte, algunos autores sostienen que si el banco central sigue una regla monetaria de tipo Laflèche (Taylor, 1993) y un régimen de tipo de cambio flexible, el tipo de cambio al igual que los precios estará regulado y determinado por la tasa de interés; es decir, por el instrumento de la política monetaria; en este caso se parte del supuesto de que se cumple la paridad de tasas de interés descubierta ${ }^{1}$; bajo este enfoque, no es necesario que el banco central tenga metas intermedias, por ejemplo: una paridad determinada del tipo de cambio nominal, ni, por tanto, que realice intervenciones en los mercados de cambios para estabilizar el tipo de cambio (Svensson, 2001; Bernanke et al. 1999).

Por otra parte, Ball (1997; 1999), Hüfner (2004), Mántey de Anguiano (2009) y otros analistas, descartan el cumplimiento de la paridad de tasas de interés en aquellas economías abiertas en las que, además de la tasa de interés, el banco central utiliza de manera regular el tipo de cambio como un segundo instrumento de política monetaria para conseguir la meta de estabilidad de precios; afirman que en tales casos, más que una regla de Taylor pura, lo que opera es un índice de condiciones monetarias con participación ponderada de la tasa de interés a corto plazo y una meta intermedia de tipo de cambio nominal.

En el presente artículo analizamos el papel del tipo de cambio en el marco de PMMI de las economías que conforman el Tratado de Libre Comercio de Norteamérica (TLCAN): Canadá, Estados Unidos y México. En particular, estimamos econométricamente el efecto de traspaso inflacionario de las fluctuaciones del tipo de cambio del dólar canadiense, el dólar estadounidense y el peso mexicano en el nivel de precios de las tres economías enunciadas durante 1990-2015, periodo en que, a partir de diferentes momentos de adopción en cada país, ha estado vigente el modelo de PMMI. Asimismo, indagamos las relaciones de causalidad Granger entre la tasa de interés y el tipo de cambio en la economía de América del Norte.

Nuestra investigación empírica revela que, en el seno del TLCAN, existe una asimetría en lo que concierne al papel del tipo de cambio en la estrategia de estabilidad de la inflación; mientras que en Canadá y

\footnotetext{
${ }^{1}$ La hipótesis de paridad descubierta de tasas de interés expresa la relación entre la tasa de interés interna y la tasa de interés externa, más las variaciones del tipo de cambio; por lo tanto, el diferencial de las tasas de interés entre dos países debería ser proporcional a la variación esperada de su tipo de cambio $\sigma^{\mathrm{e}}=\mathrm{i}-\mathrm{i}^{*}$, donde $\sigma^{\mathrm{e}}$ es la tasa esperada de variación en el tipo de cambio. Bajo el supuesto de ausencia de impuestos, riesgo y costos de transacción, el proceso de arbitraje iguala las tasas de interés entre los dos países: $r=r^{*}$.
} 
Estados Unidos el coeficiente del traspaso inflacionario es prácticamente nulo, en México su magnitud ha descendido pari passu con el abatimiento de la inflación (tal como pronostica la hipótesis de Taylor, 2000), pero aún es estadísticamente significativo, particularmente en términos relativos vis-à-vis el coeficiente observado en Canadá y Estados Unidos. Evidentemente, en el contexto de una zona de libre comercio como la que representa el TLCAN, esta discrepancia es relevante para la estabilidad tanto de la balanza de pagos como del crecimiento del producto y del empleo de la economía mexicana.

En esta investigación revisamos la bibliografía relevante sobre el efecto de traspaso de las variaciones del tipo de cambio al nivel de precios en México, Canadá y Estados Unidos; a continuación presentamos algunos hechos estilizados del comportamiento de las variables involucradas en el fenómeno del efecto de traspaso del tipo de cambio a la inflación; posteriormente, estimamos econométricamente el efecto de traspaso inflacionario y las relaciones de causalidad Granger entre la tasa de interés y el tipo de cambio en las economías de América del Norte. La última parte contiene las conclusiones que se derivan del análisis de la evidencia empírica.

\section{Revisión bibliográfica}

Diversos análisis empíricos del efecto de las variaciones del tipo de cambio al nivel de precios en Canadá, Estados Unidos y México concluyen que la magnitud del coeficiente de traspaso inflacionario disminuyó notablemente después de que los bancos centrales adoptaron el marco de PMMI.

Taylor (2000) plantea la hipótesis de que el traspaso inflacionario está en función de la historia de la inflación; presenta un modelo en el que la formación de precios depende de cambios en los costos, del precio fijado por otras empresas y de cambios en la demanda:

$$
\begin{gathered}
y_{t}=\varepsilon_{t}-\beta\left(x_{t}-p_{t}\right) \\
\sum_{\mathrm{i}=0}^{3} E_{t}\left(x_{t} y_{t-i}-2 c_{t+\mathrm{i}} y_{t+i}\right) \\
x_{t}=0.125 \sum_{\mathrm{i}=0}^{3}\left(E_{t} c_{t+\mathrm{i}}+E_{t} p_{t+\mathrm{i}+} E_{t} \varepsilon_{t+\mathrm{i}} / \beta\right) \\
c_{t}=p c_{t-1}+u_{t}
\end{gathered}
$$

Donde $y_{t}$ es la producción, $x_{t}$ es el precio del bien, $p_{t}$ es el precio medio de las mercancías diferenciadas, $\varepsilon_{t}$ es un cambio aleatorio en la demanda, $c_{t}$ es el costo de producción marginal del bien, $E_{t}$ representa el operador 
de esperanza condicional basado en la información en el periodo $t$. La ecuación 1 corresponde a la curva de demanda lineal de la empresa (la diferenciación del producto es un aspecto esencial para el consumidor); es una media de los precios fijados por las empresas en los cuatro periodos más recientes; la ecuación 2 es el beneficio esperado de empresa.

El precio óptimo (ecuación 3) se obtiene sustituyendo la ecuación 1 en la ecuación 2 y diferenciando respecto a $x_{i}$. El coeficiente 0.125 implica que un aumento de una unidad en el precio de otras empresas y en el costo marginal resulta en un incremento de una unidad en el precio óptimo de la empresa representativa; pero si sólo $c_{t}$ aumenta en una unidad, $x_{t}$ se incrementará sólo 0.5 unidades; en ninguno de los dos casos la magnitud del traspaso depende de la pendiente de la curva de demanda lineal.

La ecuación 4 muestra que el efecto sobre el precio, derivado de un aumento en $c_{t}$, depende del aumento permanente de $c_{i}$; una menor persistencia en el aumento de los costos marginales reduce el coeficiente de traspaso inflacionario, de suerte que la fijación de precios depende en gran medida de las expectativas de los movimientos en los precios y los costos futuros. Una depreciación del tipo de cambio elevará el costo de las importaciones en moneda nacional. Con base en la ecuación 3, si la depreciación es temporal (con relación a la tasa de inflación subyacente), la empresa trasladará la depreciación en forma de un $x_{t}$ más elevado; por lo tanto, menores fluctuaciones en el tipo de cambio conducirán a coeficientes de traspaso más pequeños.

Dornbusch (1976) propone un modelo dinámico que explica el fenómeno overshooting del tipo de cambio; incluye tres variables endógenas: tipo de cambio, nivel de precios y tasa de interés doméstica, y sostiene que existe una correspondencia biunívoca perfecta entre el nivel de precios y la tasa de interés. El modelo de Dornbusch supone que la velocidad de ajuste del tipo de cambio es instantánea y mucho mayor que la del nivel de precios, lo que implica que ante un cambio de una variable exógena el efecto sobre el tipo de cambio sea mayor en el corto que en el largo plazo, es decir, en el corto plazo supera al valor de equilibrio de largo plazo. En consecuencia, ante un cambio de política monetaria el mercado de divisas reaccionará exageradamente en el corto plazo, propiciando un equilibrio efímero. Sin embargo, eventualmente los precios de los bienes se ajustarán, el mercado de divisas eliminará el sobreajuste y la economía alcanzará un nuevo equilibrio de largo plazo en todos los mercados.

Goldfajn y Werlang (2000) analizan la relación entre la depreciación del tipo de cambio y la inflación para una muestra de 71 países en el periodo 1980-1998, encuentran que el coeficiente de traspaso inflacionario es sustancialmente menor en los países desarrollados respecto a los no industrializados; los factores determinantes más importantes del efecto 
de traspaso son la sobrevaluación del tipo de cambio real y la inflación inicial; las expectativas no son significativamente importantes.

Baqueiro et al. (2003) analizan 16 economías abiertas con tipos de cambio flexibles y en proceso de estabilización de precios, y prueban la hipótesis de Taylor (2000) de que el traspaso inflacionario disminuye con la estabilidad de la inflación y reduce el miedo a flotar. Bailliu y Fujii (2004) también prueban la hipótesis de Taylor mediante datos de panel de 11 países industrializados, para el periodo 1977-2001; sus resultados sugieren que el coeficiente de traspaso inflacionario se redujo tras la estabilización de la inflación en muchos países industrializados a principios del 1990.

Gagnon y Ihrig (2004) analizan 20 países industriales para el periodo 1971-2003, muestran que la correlación implícita entre los cambios de tipo de cambio y la inflación depende del régimen de política monetaria; argumentan que cuando los agentes económicos comprenden las intenciones del banco central son menos propensos a trasladar los efectos de los movimientos en el tipo de cambio a los precios. Takhtamanova (2008) analiza 14 países de la Organización para la Cooperación y el Desarrollo Económicos (OCDE), compara los coeficientes promedio de la curva de Phillips y las respuestas promedio de la dinámica de la inflación ante un choque en el tipo de cambio real; concluye que durante la década de 1990 se produjo un cambio estructural en la relación entre la inflación y el tipo de cambio real.

Jašová et al. (2016) estudiaron los efectos de la crisis financiera subprime sobre el traspaso inflacionario en 22 economías emergentes y 11 desarrolladas en el periodo 1994-2015 utilizando datos de panel dinámico; concluyeron que la repercusión de los movimientos del tipo de cambio en las economías emergentes disminuyó después de la crisis financiera debido a la disminución de la inflación, mientras que en las economías avanzadas el traspaso se ha mantenido relativamente bajo y estable, dado que niveles de transferencia generalmente bajos implican que los bancos centrales temen menos a la flotación de sus tipos de cambio.

Caselli y Roitman (2016), centrados en la existencia de no linealidades y asimetrías, mediante técnicas de proyección local, calculan el traspaso inflacionario de los tipos de cambio a los precios de 28 economías emergentes; encuentran evidencia significativa de que al considerar asimetrías y no linealidades durante episodios de depreciación mayores a 10 y 20\%, el efecto de traspaso fluctúa entre 18 y $25 \%$, mientras que para la estimación lineal es igual a $6 \%$. Caselli et al. Concluyen que el traspaso decrece con el grado de anclaje de las expectativas de inflación y ha sido relativamente moderado, dado el tamaño y la persistencia de la depreciación y debido a las mejoras de los regímenes monetarios. 
El Fondo Monetario Internacional (FMI, 2016) también realizó estimaciones empíricas (mediante el método de proyección local) del traspaso inflacionario de los tipos de cambio con base en una muestra que incluye datos mensuales - de enero de 2000 a diciembre de 2015- de 31 economías avanzadas y 31 economías de mercados emergentes; en América Latina, el traspaso es inferior a 0.3 , el de Asia emergente es cercano a 0.2 , el de las economías emergentes de Europa es de alrededor de 0.5; la variación entre países de una misma zona es sustancial. En México el traspaso estimado es de 0.07 , significativamente inferior al del resto de América Latina.

Amador et al. (2015) estiman, mediante modelos VAR, el coeficiente de traspaso inflacionario del tipo de cambio a precios para los países que operan con un marco de política monetaria de objetivos de inflación en América Latina; muestran que en el periodo 2000-2015 no hay fuerte evidencia de aumento significativo en la región; para el caso específico de México concluyen que ha disminuido en los últimos años; diversos factores contribuyeron a generar mayores presiones sobre los precios internos en cada país: aumentos de impuestos en Chile y Brasil; shocks de oferta en el precio de los alimentos en Colombia y Perú; realineamiento de los precios gubernamentales en Brasil; reformas y cambios regulatorios en México; y la inercia salarial en Uruguay y Brasil.

Santaella-Castell (2004) analiza el caso de México durante el periodo 1969-2003, considera distintos índices de precios al consumidor y productor; encuentra un vector de cointegración entre el índice de precios interno y el índice de precios externo (ajustado por el tipo de cambio); concluye que el coeficiente de traspaso inflacionario para el subperiodo 1996-2003 es menor que para el periodo 1969-2003.

Galindo y Ros (2008) analizan la experiencia de México con el régimen de metas de inflación mediante un modelo VAR; concluyen que este régimen ha permitido la reducción de la inflación y del efecto de traspaso de las variaciones del tipo de cambio a los precios (posiblemente debido a una mayor credibilidad de los bancos centrales y a la estabilización de las expectativas); la desinflación ha comportado la continua apreciación del tipo de cambio real, resultado de una política asimétrica respecto a los movimientos del tipo de cambio que contrae la actividad económica; finalmente, Galindo y Ros abogan por una política de tipo de cambio real competitivo.

Cortés (2013) también analiza el caso de México al estimar un modelo de Vectores Autorregresivos (VAR); sus resultados sugieren que el coeficiente de traspaso inflacionario disminuyó a partir de 2000, que es bajo y estadísticamente no significativo y que la depreciación de 2011 no modificó esta trayectoria. Capistrán et al. (2011) igualmente calculan el coeficiente de traspaso inflacionario en México con un modelo VAR para 
el periodo 1997-2010, utilizan los impulsos respuesta acumulados de un choque al tipo de cambio identificados mediante el método recursivo; sus resultados muestran que el traspaso inflacionario de los precios de las importaciones es completo, pero disminuye a lo largo de la cadena de distribución: el impacto sobre los precios al consumidor es menor al 20 por ciento.

Castillo et al. (2013) evalúan el traspaso inflacionario en México en el corto y largo plazos con la prueba de cointegración de Johansen y la prueba de ciclos comunes de Vahid y Engle; concluyen que ha disminuido tanto a nivel nacional como regional. Pérez-Laurrabaquio (2012) estudia el coeficiente de traspaso inflacionario en México durante el periodo 1995-2012 con base en dos modelos VAR y sus funciones impulso-respuesta; sus resultados comprueban la hipótesis de Taylor (2000).

Para el caso de Canadá, Laflèche (1997) encuentra que en el corto plazo una depreciación de la moneda da lugar a un aumento de los precios de insumos importados, lo que se traduce en mayores costos de producción. La magnitud del aumento de los costos y la velocidad con que se traslada a los consumidores depende de la participación relativa de las importaciones en la canasta de consumo, de las condiciones de demanda y las expectativas temporales de la devaluación del tipo de cambio. Bouakez y Rebei (2006) estiman un modelo dinámico y estocástico de equilibrio general; concluyen que el coeficiente de traspaso inflacionario ha sido estable, lo cual se atribuye al régimen de política monetaria.

Savoie-Chabat y Khan (2015) también analizan cuantitativamente el traspaso de las fluctuaciones del tipo de cambio a la inflación, concluyen que en Canadá la inflación ha sido impulsada por los movimientos del tipo de cambio; sin embargo, con expectativas de inflación a largo plazo ancladas cerca de la meta de inflación del Banco de Canadá se espera que el traspaso inflacionario ejerza sólo una influencia transitoria en la tasa de inflación.

Devereux et al. (2015) analizan el impacto de la estructura de mercado en la determinación del traspaso inflacionario del tipo de cambio mediante un gran conjunto de microdatos de las importaciones de Canadá; la evidencia estadística, afirman, sugiere que la estructura de mercado, el volumen de importaciones y la cuota de mercado tienen implicaciones importantes en la sensibilidad de los precios internos ante los movimientos del tipo de cambio.

Coughlin y Pollard (2000) analizan el coeficiente de traspaso inflacionario en los precios de la industria manufacturera de Estados Unidos, concluyen que, aunque es muy complicado medirlo, dado que se trata del importador global para el cual hay que estimar un indicador complejo del tipo de cambio, el efecto de traspaso es positivo pero ha disminuido notablemente en años recientes. 


\section{Hechos estilizados}

Canadá, Estados Unidos y México han transitado con diferentes velocidades de experiencias de alta inflación —incluso hiperinflación-a un escenario de inflación baja y estable. Los regímenes de inflación baja y estable propician una disminución del coeficiente de traspaso inflacionario y del miedo a la flotación libre del tipo de cambio (Baqueiro et al. 2003). Esta transición ha manifestado una evolución del tipo de cambio y las tasas de interés que amerita un escrutinio estadístico y analítico. A continuación describimos el comportamiento de estas variables en los tres países de América del Norte entre enero de 1990 y diciembre de 2015.

El tipo de cambio del dólar canadiense respecto del dólar estadounidense ha fluctuado marcadamente sobre todo a principios de los años 1990 y entre 2002 y 2009 y, luego, entre 2014 y 2015. Sin embargo, no se observa que los movimientos del tipo de cambio (tasa de crecimiento anual) se traspasen al nivel de precios (variaciones anuales del índice de precios al consumidor). Esto parece indicar que en Canadá el coeficiente de traspaso inflacionario no es significativo (gráfica 1), es decir, no parece existir un canal de transmisión del tipo de cambio a la inflación.

En el caso de Estados Unidos, la correlación entre las variaciones anuales del tipo de cambio y la inflación muestra un comportamiento similar al de Canadá: no observamos una relación directa entre ambas variables. La mayor depreciación del dólar ocurrió en el periodo inmediatamente posterior al estallido de la crisis financiera subprime. No obstante, el nivel general de precios ha tendido a la baja (gráfica 2). El

\section{Gráfica 1}

\section{Canadá: inflación y tipo de cambio}

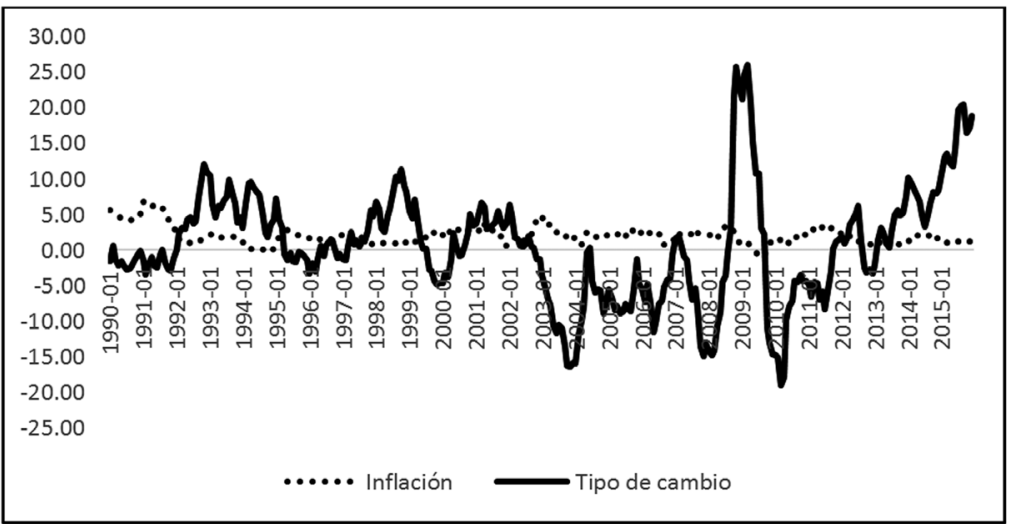

Fuente: elaboración propia con datos de la FRED (2016). 
tipo de cambio en este caso corresponde a un promedio ponderado del valor de cambio del dólar de Estados Unidos vis-à-vis las monedas de los principales socios comerciales de Estados Unidos (Zona Euro, Canadá, Japón, México, China, Reino Unido, Taiwán, Corea, Singapur, Hong Kong, Malasia, Brasil, Suiza, Tailandia, Filipinas, Australia, Indonesia, India, Israel, Arabia Saudita, Rusia, Suecia, Argentina, Venezuela, Chile

Gráfica 2

\section{Estados Unidos: inflación y tipo de cambio}

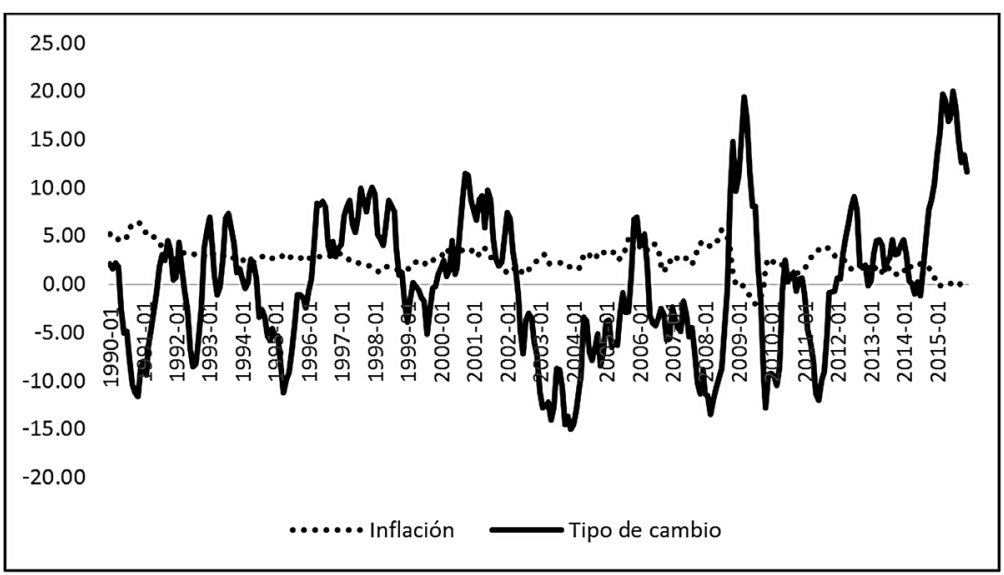

Fuente: elaboración propia con datos de la FRED (2016).

y Colombia).

En el caso de México observamos una relación distinta entre la inflación y el tipo de cambio: los movimientos de la inflación son antecedidos por devaluaciones del peso mexicano; antes de la adopción del marco de PMMI, las fluctuaciones del tipo de cambio parecen influir en el nivel general de precios con mayor fuerza que después del momento en que el Banco de México adoptó este nuevo marco de política. La devaluación de 1995 estuvo asociada claramente a una elevación del nivel general de precios; a fines de 1998 ocurrió una nueva devaluación que, de igual forma, se tradujo en un incremento de precios.

En 2008 México adoptó explícitamente un marco de PMMI; puede decirse que desde entonces el efecto de las variaciones del tipo de cambio en la inflación ha disminuido. Por ejemplo, la devaluación de 2009 generó un efecto inflacionario menor que en el pasado (gráfica 3), si bien el régimen de libre flotación del tipo de cambio conoció episodios de intervenciones esterilizadas en los mercados cambiarios para mantener la meta de inflación del Banco de México, algunos autores han interpretado este hecho como evidencia del fenómeno conocido como miedo a flotar (Calvo y Reinhart, 2000; Mántey de Anguiano, 2009). 


\section{Gráfica 3 \\ México: inflación y tipo de cambio}

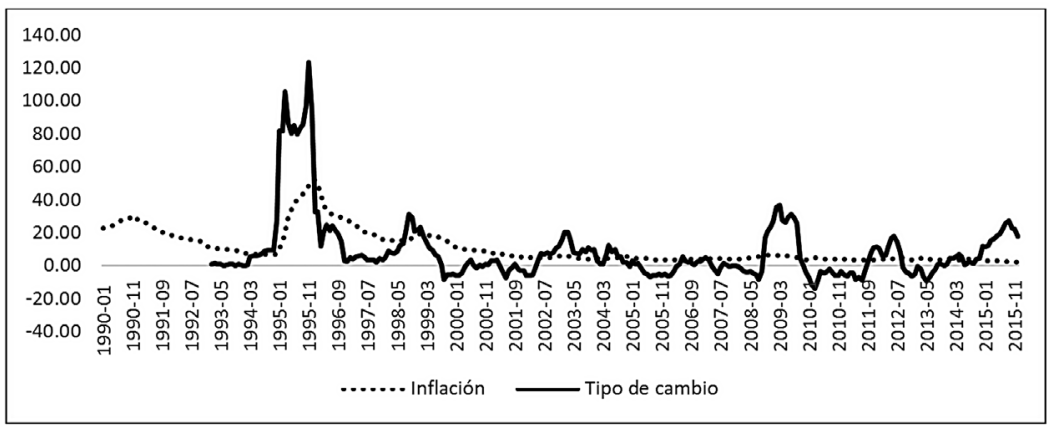

Fuente: elaboración propia con datos de Banxico (2016).

Un análisis comparativo de la evolución de los tipos de cambio, las tasas de inflación y de interés de Canadá, Estados Unidos y México revela aspectos interesantes de la convergencia económica entre las naciones de América del Norte. Dado un índice mensual del tipo de cambio nominal (base enero $1990=100$ ) para los tres países, observamos una acentuada volatilidad del peso mexicano vis-à-vis las monedas de Canadá y Estados Unidos, magnificada sobre todo después de la crisis financiera internacional de 2008 (esta relación monetaria quedó septuplicada en 2015 respecto de 1990).

El tipo de cambio del dólar canadiense respecto al estadounidense es más estable, con ligeras apreciaciones interrumpidas por los efectos de la crisis financiera subprime, la cual provocó dos periodos de ligera depreciación (01/2008-04/2009 y 01/2015 a la fecha). El tipo de cambio multilateral de Estados Unidos muestra una constante apreciación, entrecortada por un lapso de poco más de cinco años de ligera depreciación (01/1997-03/2003). Contrariamente a lo ocurrido en México y Canadá, la crisis financiera subprime derivó en un periodo (10/2007-06/2008) de fuerte apreciación del dólar estadounidense respecto a las monedas de sus principales socios comerciales, alcanzando su valor más alto en julio de 2011 (gráfica 4).

Por otra parte, la entrada en vigor del Tratado de Libre Comercio de Norteamérica (TLCAN) en 1994 y la adopción del marco de PMMI indujeron una forzada convergencia entre las tasas de inflación y de interés de las tres economías en cuestión (gráficas 5 y 6): la tasa de inflación de México ha tendido a converger más velozmente con las de Canadá y Estados Unidos que la tasa de interés.

Si bien es cierto que la brecha de tasas de interés ha disminuido desde la adopción de un modelo de política monetaria de metas de inflación en 


\section{Gráfica 4}

\section{Evolución de los tipos de cambio: Canadá, México y Estados Unidos}

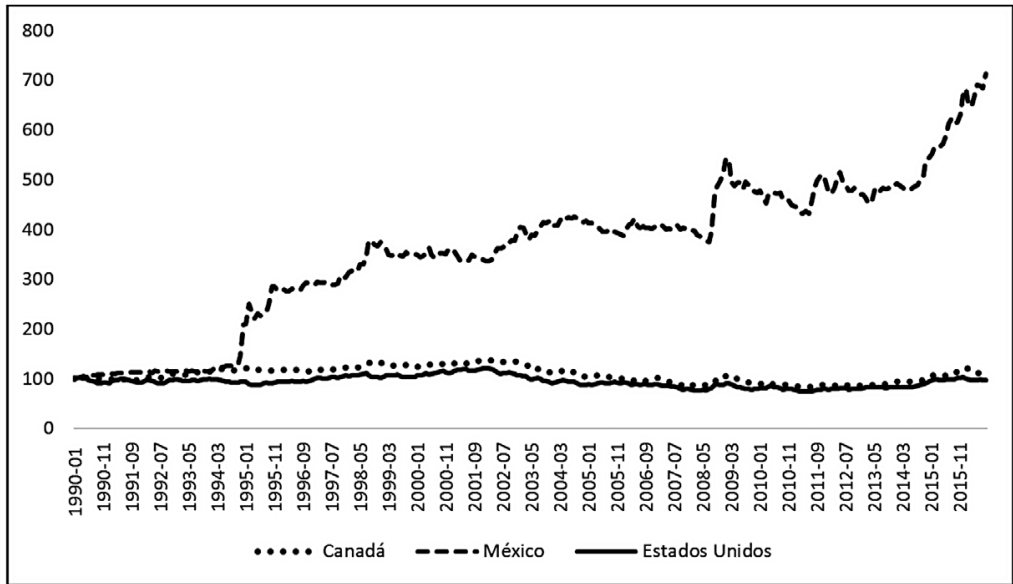

Fuente: elaboración propia con datos de la FRED (2016).

Gráfica 5

Evolución de la inflación: México, Estados Unidos y Canadá

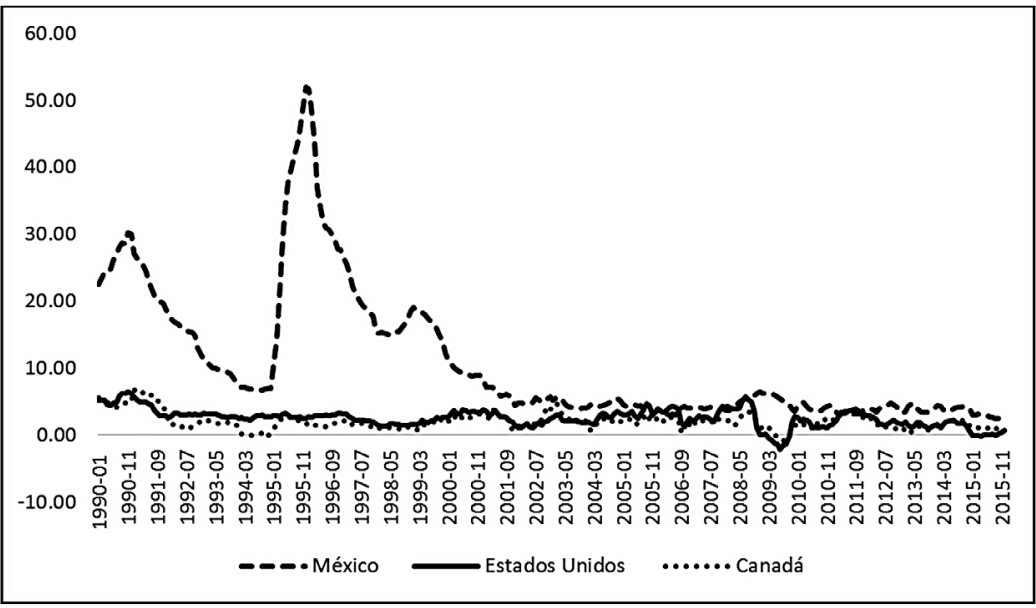

Fuente: elaboración propia con datos de la FRED (2016). 


\section{Gráfica 6}

\section{Evolución de la tasa de interés: México, Estados Unidos y Canadá}

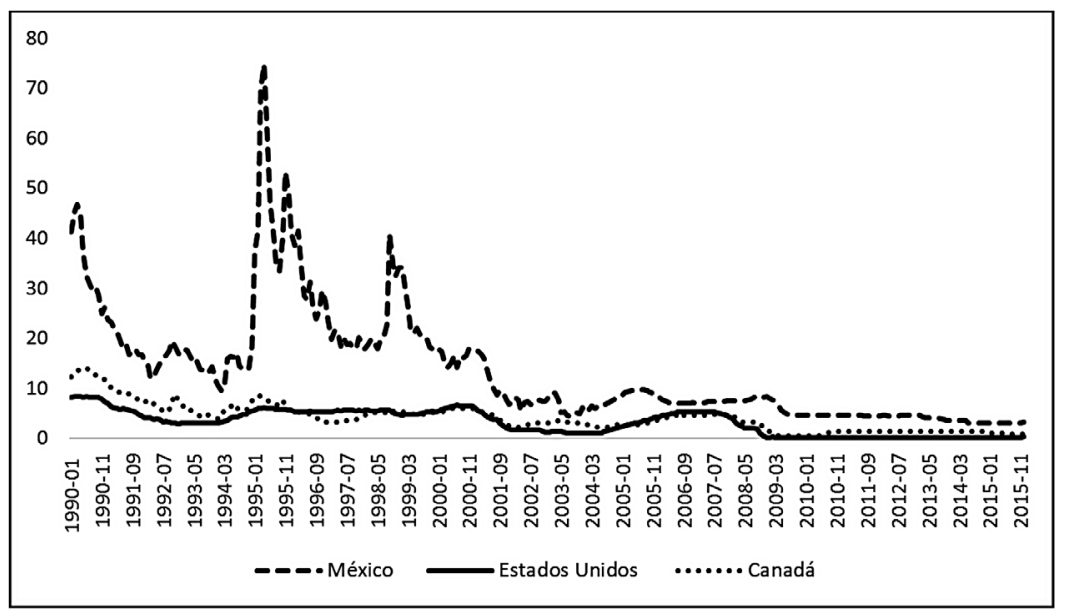

Fuente: elaboración propia con datos de la FRED (2016).

México, esta asimetría persiste; el arbitraje de tasas de interés aún es incompleto pese a la profunda liberalización e integración financiera; hecho que repercute directamente en las relaciones comerciales que sostiene con sus principales socios comerciales. En lo que sigue, presentamos una medición del efecto traspaso inflacionario del tipo de cambio a los precios de las tres economías, así como un análisis de las relaciones de causalidad entre el tipo de cambio y la tasa de interés.

\section{Análisis econométrico}

\subsection{Estimación del efecto de traspaso inflacionario}

En la estimación del efecto traspaso inflacionario empleamos datos mensuales de la FRED y del Banxico del periodo 1990-2015 de las siguientes variables: tipo de cambio de México (TCM) y Canadá (TCC); tasa de interés de México (TIM), Canadá (TIC) y Estados Unidos (TIEU); inflación de México (IM), Canadá (IC) y Estados Unidos (IEU); reservas internacionales de México (RMM), Canadá (RMC) y Estados Unidos (RIEU).

Para México y Canadá realizamos dos estimaciones con transformaciones logarítmicas, mediante mínimos cuadrados ordinarios (MCO), con el objetivo de generar información cualitativa, incluimos una variable 
dummy que captura las recesiones y periodos de inestabilidad inflacionaria más relevantes en el periodo de estudio.

Los resultados econométricos para el análisis del caso de México son consistentes con los de otros estudios similares y complementan el análisis de los hechos estilizados antes discutidos. En general, en estos estudios se asume que la elasticidad de traspaso inflacionario aumenta con el periodo de tiempo analizado; Cortes (2013) encuentra un coeficiente de elasticidad de traspaso de 0.04 en el lapso de un año y de 0.06 en cuatro ańos; Castillo et al. (2013) estiman que la elasticidad de traspaso en el periodo de paridad fija es de 0.01 y de 0.02 para el periodo de libre flotación. Capistrán et al. (2011) estiman para el corto plazo (seis meses) una elasticidad del traspaso inflacionario de 0.04 , la elasticidad se incrementa a 0.4 si el plazo es de 24 meses, y para el largo plazo estiman que es de 0.16. Mántey de Anguiano (2012) estima que la elasticidad del traspaso inflacionario del tipo de cambio a los precios es de 0.20 para los países en desarrollo y de 0.04 en el caso de los países industrializados.

En nuestro análisis encontramos que el coeficiente de elasticidad de traspaso del tipo de cambio a los precios en México es de 0.125 para el periodo de 1990 a 2015. Nuestro estudio es a largo plazo y confirma la persistencia de un efecto de traspaso inflacionario (cuadro 1), de ahí las eventuales intervenciones esterilizadas del Banco de México.

\section{Cuadro 1}

\section{México: medición del efecto de traspaso inflacionario}

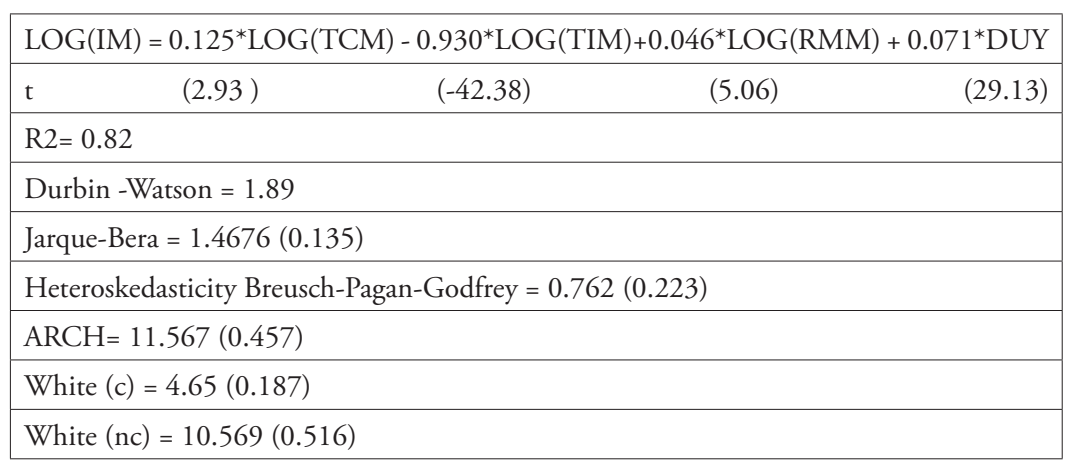

Fuente: elaboración propia.

El coeficiente de la tasa de interés es negativo, es decir, existe una relación inversa entre la inflación y la tasa de interés: si la tasa de interés aumenta la inflación baja, tal como postula el marco de PMMI. Sin embargo, el valor del coeficiente de la tasa de interés es pequeño, la elasticidad es baja porque la inflación es muy cercana a la meta; mientras no se aleje de la meta, el banco central no tiene incentivos para realizar 
movimientos de la tasa de interés. El parámetro de las reservas internacionales tiene signo positivo pero su bajo valor indica que la tenencia de reservas no es determinante en el control de la inflación. El abultado portafolio de reservas internacionales se explica por la necesidad de defender la paridad cambiaria y por la importancia y relación de la estabilidad del tipo de cambio con la inflación.

Las pruebas confirman que los resultados de la estimación son estadísticamente satisfactorios; el cuadro 1 contiene, además de los valores de estimados de los coeficientes, las t estadísticas de significancia, las pruebas de normalidad, autocorrelación y homocedasticidad. En la figura 1 observamos que la prueba de Cusum muestra cambio estructural para los años 1995 y 2007. Sin embargo, en ambos casos existe corrección en los siguientes ańos, lo cual elimina el cambio estructural en lo general; la prueba de Cusum Q no revela indicios de cambio estructural.

En el caso de Canadá, con la estimación mediante MCO no encontramos evidencia de un efecto de traspaso de las fluctuaciones del tipo de cambio al nivel general de precios, el parámetro es pequeño y negativo. Esta parece ser la razón que explica que el banco central de Canadá permita la libre fluctuación del dólar de ese país.

El resultado de la estimación confirma que existe una relación inversa entre la inflación y la tasa de interés: ante un aumento de la inflación, tal como lo plantea una PMMI, el Banco de Canadá responde con un incremento de la tasa de interés; el bajo valor del coeficiente se debe a que, igual que en México, el nivel de inflación observada es muy cercano a la meta de inflación del banco central. De ahí la baja elasticidad, incluso menor en Canadá que en México; el parámetro de las reservas internacionales indica una relación positiva entre éstas y la inflación; la elasticidad es baja pero mayor que en el caso de México.

Figura 1

\section{México: prueba Cusum y Cusum $Q$}

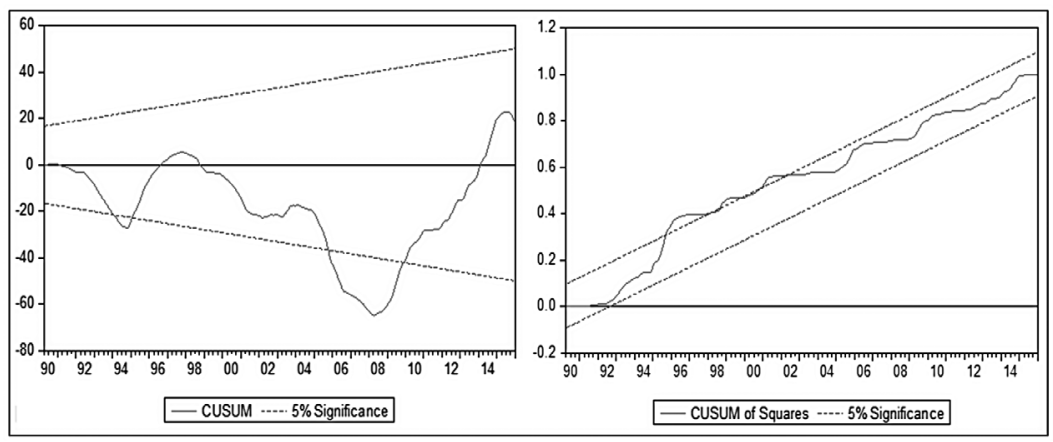

Fuente: elaboración propia. 
Los valores de los parámetros estimados y las pruebas que confirman que los resultados de la estimación son estadísticamente satisfactorios para el caso de Canadá (cuadro 2). La figura 2 muestra los resultados de la prueba de Cusum de cambio estructural y la prueba de Cusum Q; ésta es la más estricta y no presenta indicios de cambio estructural; por lo tanto se plantea que la estimación es correcta.

\section{Cuadro 2}

\section{Canadá: Medición del efecto de traspaso inflacionario}

\begin{tabular}{|l|}
\hline LOG $(\mathrm{IC})=-0.034 * \mathrm{LOG}(\mathrm{TIC})-0.099 * \mathrm{LOG}(\mathrm{TCC})+0.187 *$ LOG $(\mathrm{RMC})+0.048 * \mathrm{DUC}$ \\
\hline $\mathrm{t} \quad(-27.088) \quad(-14.576)$ \\
\hline $\mathrm{R}^{2}=.98$ \\
\hline Durbin -Watson $=1.70$ \\
\hline Jarque - Bera $=0.026(0.986)$ \\
\hline Heteroskedasticity Breusch-Pagan-Godfrey $=3.549(0.470)$ \\
\hline ARCH= $17.282(0.139)$ \\
\hline White $(\mathrm{c})=5.511(0.257)$ \\
\hline White $(\mathrm{nc})=16.419(0.088)$ \\
\hline
\end{tabular}

Fuente: elaboración propia.

La estimación para Estados Unidos requirió la inclusión de valores autoregresivos para las variables IEU y TCEU a fin de corregir el problema de autocorrelación. El resultado de la estimación mediante $\mathrm{MCO}$ refleja la irrelevancia de los movimientos del tipo de cambio en el desempeño de la inflación. A diferencia de México y Canadá, en Estados Unidos el resultado no confirma el mecanismo de transmisión de la PMMI (cuadro

Figura 2

Canadá: prueba Cusum y Cusum $Q$

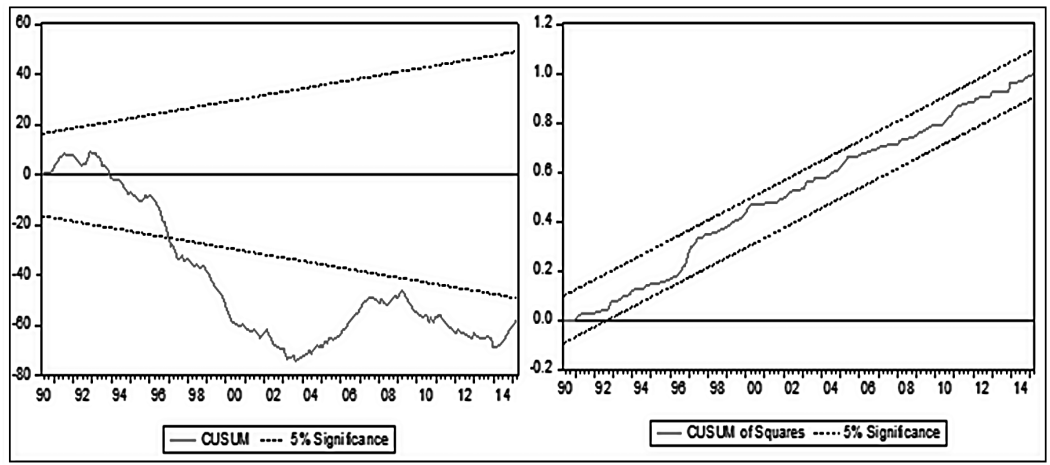

Fuente: elaboración propia. 
3): no parece que exista una relación inversa entre la inflación y la tasa de interés. Como es sabido, después del estallido de la crisis financiera subprime, la FED abandonó la política monetaria de regla de Taylor y adoptó un marco de política monetaria no convencional (Bernanke, 2013; 2015) donde, mediante la compra masiva de activos y flexibilización de crédito, la hoja de balance de la FED sustituyó a la tasa de interés de los fondos federales como instrumento predilecto de la política monetaria.

Las pruebas que confirman que los resultados de la estimación son estadísticamente correctos para Estados Unidos se presentan en el cuadro 3. La figura 3 muestra las pruebas de Cusum y Cusum $Q$, ambas descartan la existencia de cambio estructural.

\section{Cuadro 3}

Estados Unidos: medición del efecto de traspaso inflacionario

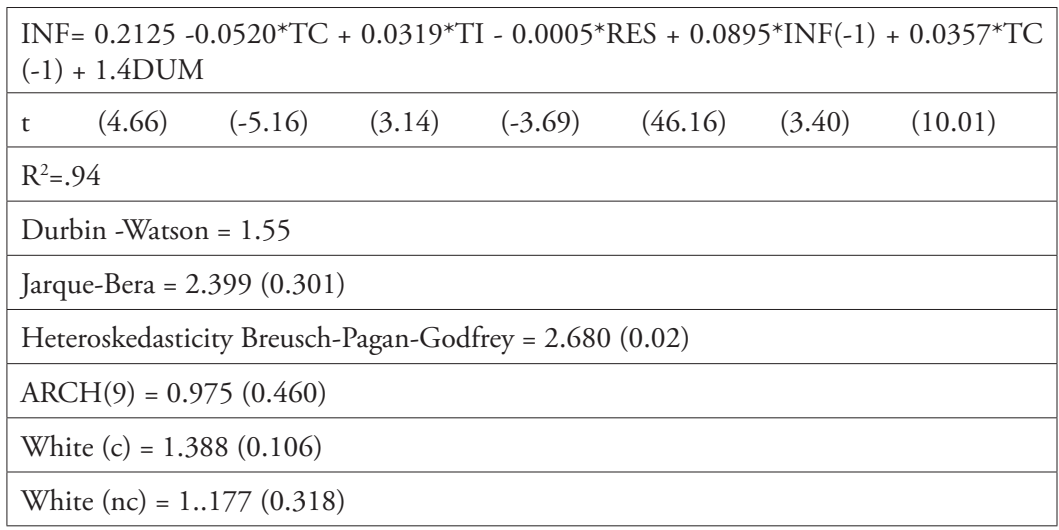

Fuente: elaboración propia.

El hecho de que el coeficiente de traspaso inflacionario en México es mayor que en Canadá y Estados Unidos, a pesar de la disminución y estabilización de la inflación, significa una asimetría adversa para la economía mexicana cuya macroeconomía se caracteriza por un aparente conflicto (trade-off) del papel dicotómico del tipo de cambio: el modelo de crecimiento exportador (con alto contenido de insumos importados) requiere un tipo de cambio competitivo y el modelo de metas de inflación exige un tipo de cambio no competitivo.

\subsection{Análisis de causalidad entre la tasa de interés y el tipo de cambio}

La hipótesis de que la tasa de interés determina al tipo de cambio es parte sustancial del marco de PMMI. En consecuencia, la tasa de interés regula los precios y la dinámica del tipo de cambio. A continuación, analizamos 
Figura 3

\section{Estados Unidos: prueba Cusum y Cusum $Q$}

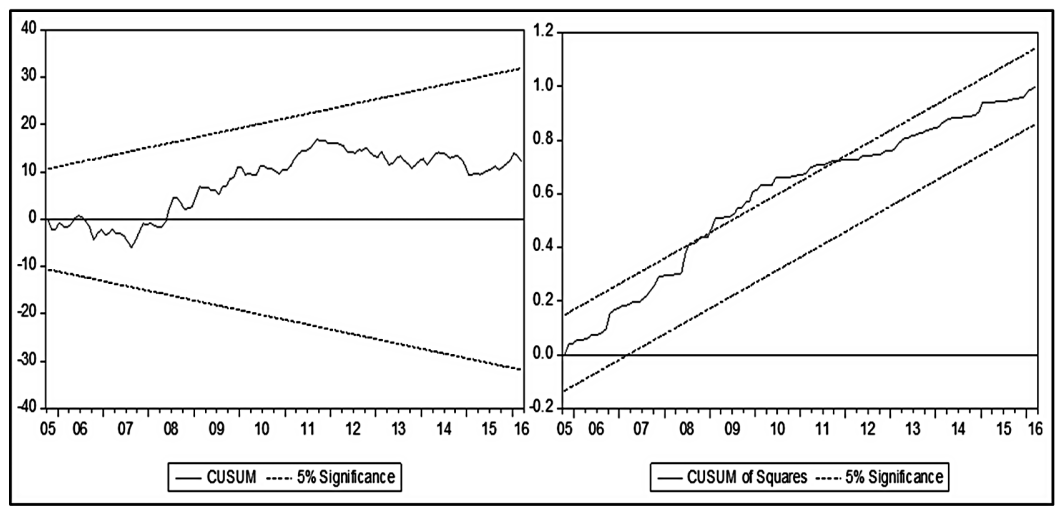

Fuente: elaboración propia.

empíricamente la relación de causalidad entre estas dos variables, la tasa de interés y el tipo de cambio de las tres economías que conforman el TLCAN. Los cuadros 4, 5 y 6 contienen los resultados de las pruebas de causalidad Granger realizadas.

En el caso de México, la hipótesis nula de no causalidad del TCM hacia la TIM se rechaza en el segundo y el tercer rezagos; el tipo de cambio causa a la tasa de interés. Es decir el bloque de los valores rezagados del tipo de cambio permite mejorar el pronóstico de la tasa de interés (cuadro 4).

Las pruebas de causalidad de Granger en el caso de Canadá revelan que no existe una relación de causalidad entre entre el tipo de cambio y la tasa de interés (cuadro 5). En Estados Unidos la tasa de interés causa al tipo de cambio en el segundo, quinto y sexto rezagos (cuadro 6).

Estas relaciones de causalidad entre la tasa de interés y el tipo de cambio confirman la asimetría antes aludida entre las políticas monetarias de Canadá, Estados Unidos y México; mientras en Estados Unidos se cumple la hipótesis de causalidad de la tasa de interés al tipo de cambio, en Canadá no existe esa relación y en México la relación es en sentido opuesto: el tipo de cambio causa a la tasa de interés.

El uso de la política cambiaria como estrategia de desarrollo, partiendo de la hipótesis de miedo a flotar, ha demostrado que los tipos de cambio depreciados inducen un mayor crecimiento a través de la profundización del ahorro interno y la acumulación de capital (Levy-Yeyati y Sturzenegger, 2007); la evidencia empírica de este trabajo muestra que si bien México no utiliza el tipo de cambio como herramienta de estabilización, lo hace de forma discrecional. 


\section{Cuadro 4}

\section{Pruebas de causalidad entre la tasa de interés y el tipo de cambio de México}

\begin{tabular}{lcccc}
\hline $\begin{array}{c}\text { Causalidad de Granger } \\
\text { Hipótesis Nula }\end{array}$ & \multicolumn{2}{c}{ TIM no causa TCM } & \multicolumn{2}{c}{ TCM no causa TIM } \\
\cline { 2 - 5 } & Estadistico F & Probabilidad & Estadistico F & Probabilidad \\
\hline Rezago 1 & 2.01 & 0.15 & 2.07 & 0.15 \\
Rezago 2 & 1.67 & 0.18 & $\mathbf{3 . 3 4}$ & $\mathbf{0 . 0 3}$ \\
Rezago 3 & 1.58 & 0.19 & $\mathbf{3 . 1 8}$ & $\mathbf{0 . 0 2}$ \\
Rezago 4 & 1.17 & 0.32 & 1.54 & 0.19 \\
Rezago 5 & 0.91 & 0.47 & 0.86 & 0.50 \\
Rezago 6 & 0.63 & 0.69 & 0.57 & 0.74 \\
\hline
\end{tabular}

Fuente: elaboración propia.

Cuadro 5

Pruebas de causalidad entre la tasa de interés y el tipo de cambio de Canadá

\begin{tabular}{lcccc}
\hline Causalidad de Granger & \multicolumn{2}{c}{ TIC no causa TCC } & \multicolumn{2}{c}{ TCC no causa TIC } \\
\cline { 2 - 5 } Hipótesis Nula & Estadistico F & Probabilidad & Estadistico F & Probabilidad \\
\hline Rezago 1 & 0.06 & 0.79 & 0.70 & 0.40 \\
Rezago 2 & 0.002 & 0.99 & 0.63 & 0.53 \\
Rezago 3 & 0.094 & 0.96 & 1.86 & 0.13 \\
Rezago 4 & 0.12 & 0.97 & 1.87 & 0.11 \\
Rezago 5 & 0.50 & 0.76 & 1.63 & 0.14 \\
Rezago 6 & 0.40 & 0.87 & 1.74 & 0.11 \\
\hline
\end{tabular}

Fuente: elaboración propia.

\section{Cuadro 6}

Pruebas de causalidad entre la tasa de interés y el tipo de cambio de Estados Unidos

\begin{tabular}{ccccc}
\hline $\begin{array}{c}\text { Causalidad de Granger } \\
\text { Hipótesis Nula }\end{array}$ & \multicolumn{2}{c}{ TIEU no causa TCEU } & \multicolumn{2}{c}{ TCC no causa TIC } \\
& Estadistico F & Probabilidad & Estadistico F & Probabilidad \\
\hline Rezago 1 & 0.39 & 0.53 & 0.06 & 0.79 \\
Rezago 2 & $\mathbf{2 . 9 5}$ & $\mathbf{0 . 0 5}$ & 0.13 & 0.87 \\
Rezago 3 & 1.82 & 0.14 & 0.44 & 0.71 \\
Rezago 4 & 1.39 & 0.23 & 0.90 & 0.46 \\
Rezago 5 & $\mathbf{2 . 3 1}$ & $\mathbf{0 . 0 4}$ & 0.66 & 0.64 \\
Rezago 6 & $\mathbf{2 . 5 0}$ & $\mathbf{0 . 0 2}$ & 0.86 & 0.51 \\
\hline
\end{tabular}

Fuente: elaboración propia. 
Levy-Yeyati y Sturzenegger (2009) consideran que la elección de un régimen de tipo de cambio no sólo tiene una implicación directa en la evolución de las variables nominales clave de la economía, también la tiene en la volatilidad de la producción y del sector financiero, lo cual, a su vez, influye en el crecimiento del producto y la distribución del ingreso.

\section{Conclusión}

En el presente artículo hemos realizado un análisis comparativo del efecto de traspaso inflacionario de las variaciones del tipo de cambio al nivel de precios en las economías de Canadá, Estados Unidos y México para el periodo 1990-2015. El patrón de comportamiento de las tasas de interés, de inflación y de los tipos de cambio respectivos permite identificar algunos hechos estilizados que revelan una alta correlación estadística meritoria de un escrutinio más detallado, a lo cual procedimos en la estimación econométrica del efecto de traspaso inflacionario y las relaciones de causalidad Granger entre la tasa de interés y el tipo de cambio en las economías de América del Norte.

En el caso de Canadá no encontramos evidencia de un efecto de traspaso de las fluctuaciones del tipo de cambio a la inflación, el parámetro es pequeño y negativo. El Banco de Canadá, por ello, dispone de más grados de libertad en el manejo de su política monetaria y de tipo de cambio; el Banco de Canadá no tiene miedo a flotar.

En el caso de Estados Unidos, nuestro análisis econométrico nos conduce a concluir que los movimientos del tipo de cambio son inocuos para el desempeño de la inflación. Más aún, no parece que exista una relación inversa entre la inflación y la tasa de interés; la FED llevó la tasa de interés al límite cero después del estallido de la crisis financiera que tuvo su epicentro en el mercado de las hipotecas subprime, posteriormente abandonó la política monetaria convencional y adoptó un marco de política monetaria no convencional centrada en la compra masiva de activos y la flexibilización de crédito. A diferencia de Canadá y Estados Unidos, en México el coeficiente de elasticidad de traspaso del tipo de cambio a los precios en México es positivo (0.125); en el largo plazo persiste un efecto de traspaso inflacionario

Asimismo, nuestro análisis de las relaciones de causalidad entre la tasa de interés y el tipo de cambio revela una segunda asimetría adversa para México, además de la referida al coeficiente de pass-through: en Estados Unidos la tasa de interés determina al tipo de cambio, en Canadá no existe relación entre ambas variables y en México el tipo de cambio causa a la tasa de interés. 
El análisis del impacto del tipo de cambio en la inflación revela implicaciones serias para México; en una economía abierta la estabilidad del nivel de precios es muy importante, estabilidad que depende significativamente de la magnitud del coeficiente de traspaso de las variaciones del tipo de cambio a la inflación. Este coeficiente difiere en los tres países analizados, su dimensión está asociada a la estabilidad relativa de cada moneda: el dólar norteamericano es la moneda de reserva mundial, el dólar de Canadá es una moneda relativamente fuerte y el peso mexicano es más vulnerable y volátil. La existencia de un efecto de traspaso inflacionario y la asimetría en la dirección de causalidad entre el tipo de cambio y la tasa de interés de cada país implican que el tipo de cambio desempeña un papel diferente en el marco de política monetaria de cada una de las economías en cuestión.

\section{Agradecimiento}

Se agradece el apoyo del programa de Becas Posdoctorales DGAPA, UNAM.

\section{Fuentes consultadas}

Amador, Javier, Enestor Dos Santos, Fabián García, Hermann González, Francisco Grippa, Juan Manuel Manías y Cecilia Posada (2015), “¿Ha aumentado el pass-through del tipo de cambio a precios en América Latina?”, Observatorio Económico Latam-BBVA Research, Bilbao, España, pp. 1-9, <https://www.bbvaresearch. com/wp-content/uploads/2015/10/Watch-passthorugh-v4_ maq-K.pdf>, 10 de marzo de 2017.

Bailliu Jeannine y Eiji Fujii (2004), "Exchange rate, pass-through and the inflation environment in industrialized countries: an empirical investigation", documento de trabajo núm. 2004-21, Banco de Canadá, Ottawa, Canadá.

Ball, Laurence (1997), "Efficient rules for monetary policy”, documento de trabajo núm. 5952, National Bureau of Economic Research, Massachusetts, Estados Unidos de América. 
Ball, Laurence (1999), "Policy rules for open economies", documento de trabajo núm. 6760, National Bureau of Economic Research, Massachusetts, Estados Unidos de América.

Banxico (Banco de México) (2016), "Banco de México, estadísticas, política monetaria e inflación”, Banxico, Ciudad de México, México, <http://www.banxico.org.mx/SieInternet/consultarDirectorioInternetAction. do?sector $=8 \&$ accion $=$ consultarCuadroA nalitico\&idCuadro=CA55\&locale $=$ es $>, 1$ de junio 2016.

Baqueiro Armando, Alejandro Díaz de León-Carrillo y Alberto TorresGarcía (2003), “¿Temor a la flotación o a la inflación? La importancia del 'traspaso' del tipo de cambio a los precios", documento de investigación núm. 2003-02, Dirección General de Investigación Económica Banxico, Ciudad de México, México.

Bernanke, Ben Shalom (2013), The federal reserve and the financial crisis, Princeton University Press, Princeton, Estados Unidos de América.

Bernanke, Ben Shalom (2015), The courage to act: a memoir of a crisis and its aftermath, W. W. Norton and Co., Nueva York, Estados Unidos de América.

Bernanke, Ben, Thomas Laubach, Frederic Mishkin y Adam Simon Posen (1999), Inflation targeting: lessons from the international experience, Princeton University Press, Princeton, Estados Unidos de América.

Bouakez, Hafedh y Nooman Rebei (2006), "Has exchange rate passthrough really declined in Canada?", documento de trabajo núm. 2005-29, Banco de Canadá, Montreal, Canadá.

Calvo, Guillermo y Carmen Reinhart (2000), "Fear or floating", documento de trabajo núm. 7993, National Bureau of Economic Research, Massachusetts, Estados Unidos de América.

Capistrán, Carlos, Raúl Ibarra-Ramírez y Manuel Ramos-Francia (2011), "El traspaso de movimientos del tipo de cambio a los precios: un análisis para la economía mexicana", documento de trabajo núm. 2011-12, Banxico, Ciudad de México, México.

Caselli, Francesca y Agustín Roitman (2016), "Non-linear exchange pass-through in emerging markets", documento de trabajo 
WP/16/1, Departamento Europeo, Fondo Monetario Internacional, Washington, Estados Unidos de América.

Castillo, Ramón; Varela, Rogelio y Juan Manuel Ocegueda (2013), “Traspaso del tipo de cambio al nivel de precios: un análisis regional", Economía, Sociedady Territorio, XIII (43), El Colegio Mexiquense, A. C., Toluca, México, pp. 675-695.

Cortés, Josué Fernando (2013), "Estimación del traspaso del tipo de cambio a los precios en México", Monetaria, Centro de Estudios Monetarios Latinoamericanos, Ciudad de México, México, pp. 311-344.

Coughlin, Cletus y Patricia Pollard (2000), "Exchange rate pass-through U. S. manufacturing: exchange rate index choice and asymmetry issues", documento de trabajo núm. 2000-022, Federal Reserve Bank of St. Louis, Missouri, Estados Unidos de América.

Devereux, Michael, Wei Dong y Ben Tomlin (2015), "Exchange rate pass-through, currency of invoicing and market share", documento de trabajo núm. 2015-31, Banco de Canadá, Ottawa, Canadá.

Dornbusch, Rudiger (1976), "Expectations and exchange rate dynamics", Journal of political economy, 84 (6), The University Chicago of Prees, Chicago, Estados Unidos de América, pp. 1161-1176.

FMI (Fondo Monetario Internacional) (2016), "Perspectivas económicas: Las Américas administrando transiciones y riesgos”, FMI/Estudios Económicos y Financieros, Washington, Estados Unidos de América.

FRED (Federal Reserve Economic Data) (2016), "Consumer price index: total, all items for Canada, consumer price index: total all items for the United States, consumer price index: total, all items for Mexico, effective federal funds rate, immediate rates: less than 24 hours: call money/interbank rate for Mexico, immediate rates: less than 24 hours: call money/interbank rate for Canada, trade weighted U.S. dollar index: major currencies, US dollar to national currency spot exchange rate for Mexico, US dollar to national currency spot exchange rate for Canada", Federal Reserve Bank of St. Louis, Missouri, Estados Unidos de América, <https://fred. stlouisfed.org>, 10 de junio de 2016. 
Gagnon, Joseph y Jane Ihrig (2004), "Monetary policy and exchange rate pass-through", Board of Governors of the Federal Reserve System, Washington, Estados Unidos de América, <https://www.federalreserve.gov/pubs/ifdp/2001/704/ifdp704r.pdf>, 25 de septiembre de 2016.

Galindo, Luis Miguel y Jaime Ros (2008), "Alternatives to inflation targeting in Mexico", International Review of Applied Economics, 22 (2), Taylor and Francis Group, Nueva York, Estados Unidos de América, pp. 201-214.

Goldfajn Ilan y Sergio Werlang (2000), "The pass-through from depreciation to inflation: a panel study", documento de trabajo, serie 5, Banco Central de Brasil, Brasilia, Brasil, <https://www.bcb. gov.br/pec/wps/ingl/wps05.pdf>, 27 de septiembre de 2016.

Hüfner, Felix (2004), Foreign exchange intervention as a monetary policy instrument: evidence for inflation targeting countries, PhysicaVerlag Heidelberg, Berlin, Alemania.

Jašová, Martina, Richhild Moessner y Előd Takáts (2016), “Exchange rate pass-through: what has changed since the crisis?", documento de trabajo núm. 583, Banco de Pagos Internacionales, Departamento Monetario y Económico, Basel, Suiza.

Laflèche, Thérèse (1997), "The impact of exchange rate movements on consumer prices", Bank of Canada Review, Ottawa, Canadá.

Levy-Yeyati, Eduardo y Federico Sturzenegger (2009), "(The effect of) monetary and exchange rate policies (on development)", documento de trabajo núm. 03/2009, Centro de Investigación en Finanzas, Escuela de Negocios, Universidad Torcuato Di Tella, Buenos Aires, Argentina.

Levy-Yeyati, Eduardo y Federico Sturzenegger (2007), "Fear of appreciation”, documento de trabajo núm. 07-047, Kennedy School of Government, Harvard University, Massachusetts, Estados Unidos de América.

Mántey de Anguiano, Guadalupe (2012), La política de la banca central en la teoría y en la práctica, Organización de las Naciones Unidas, 
Departamento de Economía-Comisión Económica para América Latina, Ciudad de México, México.

Mántey de Anguiano, Guadalupe (2009), "Intervención esterilizada en el mercado de cambios en un régimen de metas de inflación: la experiencia de México", Investigación Económica, LXVIII número especial, UNAM, Ciudad de México, México, pp. 47-78.

Pérez-Laurrabaquio, Oscar (2012), "El traspaso del tipo de cambio a los precios: una aproximación al caso de México", Economía Informa, núm. 376, UNAM/Facultad de Economía, Ciudad de México, México, pp. 61-73.

Santaella-Castell, Julio Alfonso (2004), "El traspaso inflacionario del tipo de cambio y la paridad del poder de compra: la experiencia mexicana”, XV Certamen Permanente de Investigación sobre Temas de Interés para la Banca Central Doctor Manuel Noriega Morales, edición 2003-2004, Guatemala, Guatemala, <http://www. banguat.gob.gt/publica/banca/047200402/002.pdf>, $01 \mathrm{de}$ octubre de 2016.

Savoie-Chabot, Laurence y Mikael Khan (2015), "Exchange rate passthrough to consumer prices: theory and recent evidence", document de investigación núm. 2015-9, Banco de Canadá, Ottawa, Canadá.

Svensson, Lars (2001), "Independent review of the operation of monetary policy in New Zealand: Report to the Minister of Finance", Reportes del tesoro de Nueva Zelanda, núm. 1, Sydney, Nueva Zelanda.

Takhtamanova, Yelena (2008), "Understanding changes in exchange rate pass-through", documento de trabajo núm. 2008-13, Federal Reserve Bank of San Francisco, San Francisco, Estados Unidos de América, <http://www.frbsf.org/economic-research/files/ wp08-13bk.pdf>, 29 de septiembre de 2016.

Taylor, John Brian (2000), "Low inflation, pass-through, and the pricing power of firms", European Economic Review, 44 (7), Elsevier, Amsterdam, Holanda, pp. 1389-1408. 
Taylor, John Brian (1993), "Discretion versus policy rules in practice", Carnegie-Rochester Conference Series on Public Policy, vol. 39, Elsevier, Amsterdam, Holanda, pp.195-214.

Recibido: 15 de diciembre de 2016. Corregido: 29 de mayo de 2017. Aceptado: 11 de agosto de 2017.

Ignacio Perrotini-Hernández. Doctor en Economía por la Universidad Nacional Autónoma de México y The Graduate Faculty of The New School For Social Research, Nueva York. Actualmente es profesor de la División de Estudios de Posgrado de la Facultad de Economía y Coordinador del Posgrado de Economía de la UNAM. Es miembro del Sistema Nacional de Investigadores, nivel III. Es traductor especializado de libros del Fondo de Cultura Económica y Consejero Ciudadano del Consejo de Evaluación del Desarrollo Social de la Ciudad de México. Sus líneas de investigación actuales son: Crecimiento y desarrollo económico, Teoría y política monetaria contemporáneas y Nuevos desarrollos en teoría macroeconómica. Algunas de sus publicaciones recientes son: "Estabilidad del crecimiento de largo plazo en los estados de la República Mexicana: nueva evidencia de pruebas de estacionariedad en panel con rupturas estructurales", Investigación Económica, LXXV (297), Universidad Nacional Autónoma de México, Ciudad de México, México, pp. 73-102 (2016); en coautoría, "Los fundamentales, las posiciones netas de los especuladores y el tipo de cambio en Brasil", Problemas del Desarrollo, 47 (186), Universidad Nacional Autónoma de México, Ciudad de México, México, pp. 161-190 (2016); en coautoría “The Fed's path to normalcy and the puzzling natural rate of interest: a comment on Armando Sánchez paper", Investigación Económica, LXXV (296), Universidad Nacional Autónoma de México, Ciudad de México, México, pp. 27-35 (2016); y "La reserva federal, la crisis y la política monetaria no convencional", Contaduría y Administración, 2 (60), Universidad Nacional Autónoma de México, Ciudad de México, México, pp. 250-271 (2015).

Ruth Ortiz-Zarco. Doctora en ciencias económicas por el Instituto Politécnico Nacional, México. Actualmente es pos-doctorante por parte de la Dirección de Formación Académica y el Departamento de Fortalecimiento Académico de la Universidad Nacional Autónoma de México; adscrita a la División de Estudios de Posgrado de la Facultad de Economía. Es miembro del Sistema Nacional de Investigadores, nivel candidato. Su línea de investigación actual es la política monetaria. Entre sus últimas publicaciones destacan: "Crisis financiera y comercio internacional: un 
análisis para los países de la OCDE”, Actas de la XVIII Reunión de economía mundial, Sociedad de Economía Mundial-Colegio de Málaga, Málaga, España, pp. 902-918 (2016), "Impacto del desarrollo del sistema financiero en el comercio de los países que integran la OCDE”, Revista Eseconomía. XI (44), Universidad Nacional Autónoma de México, Ciudad de México, México, pp. 35-62 (2016) e "Innovación tecnológica en el pensamiento económico", Revista de Administración y Finanzas, 2 (5), ECORFAN-Bolivia, Bolivia, pp. 925-941 (2015). 